\title{
EVIDÊNCIAS TEÓRICAS SOBRE A CONTRIBUIÇÃO DA GESTÃO POR PROCESSOS PARA A INOVAÇÃO AMBIENTAL
}

Sarah de Oliveira Silva dos Santos

Mestranda em Administração de Organizações FEARP/ USP. saraholilis@gmail.com

Lara Bartocci Liboni

Doutora em Administração pela FEARP/USP.

Professora do departamento de Administração de Organizações FEARP/USP. lara.liboni@gmail.com

\section{Silvia Inês Dallavalle de Pádua}

Pós-Doutora em Administração pela FEB/UNESP.

Professora do departamento de Administração de Organizações FEARP/USP. dallavalle.silvia@gmail.com

Perla Calil Pongeluppe Wadhy Rebehy

Doutora em Administração pela FEARP/USP.

Professora do departamento de Administração de Organizações FEARP/USP.

perla@netsite.com.br

\section{RESUMO}

O surgimento de uma nova ordem de mudança econômica tem levado as organizações a incluírem práticas de sustentabilidade em gestão, processos e produtos. Neste contexto, as organizações precisam de medidas de desempenho ambiental para conduzirem estratégias de longo prazo e a inovação ambiental, ou environmental innovation, é a chave para a sustentabilidade, tanto em termos econômicos quanto ecológicos. O consenso sobre a necessidade de desenvolvimento de práticas ambientais nas organizações, muitas vezes, porém, ignora a otimização das operações como fator crucial para reduzir os impactos negativos de emissões de poluentes. Esta otimização das operações e processos pode ser alcançada com abordagem de gestão focada em processos, Business Process Management (BPM). A proposição deste artigo é de que a gestão por processos (BPM), com seu foco no entendimento, modelagem, melhoria e otimização de processos de negócios, pode contribuir com a inovação ambiental. Por meio de uma revisão sistemática da literatura, buscou-se, por meio deste trabalho, evidências para esta proposição. Os resultados encontrados são que há evidências de relações positivas entre os dois temas e, pelo número reduzido de trabalhos que correlacionem o tema, é possível dizer que há espaço para desenvolvimento de pesquisa nessa área.

Palavras-chave: Inovação ambiental, Gestão por processos; Otimização de produtos e processos; Sustentabilidade.

\section{THEORETICAL EVIDENCE FOR THE CONTRIBUTION OF PROCESS MANAGEMENT FOR ENVIRONMENTAL INNOVATION}

\section{ABSTRACT}

The emergence of a new order of economic change has led organizations to incorporate sustainability practices in management processes and products. Organizations need environmental performance measures to drive long-term strategies and environmental innovation is the key to sustainability, both economically and environmentally. The consensus about the need for development of environmental practices in organizations, however, ignores the optimization of operations as a crucial factor to reduce the negative impacts of emissions. This optimization of operations and processes can be achieved with Business Process Management (BPM). The purpose of this article is that the BPM, with its focus on understanding, modeling, improvement, and optimization of business processes can contribute to environmental innovation. We used a systematic literature review to seek evidence for this proposition. We found evidence of positive relationships between the two issues, and the few studies that correlate the theme tell us that there is room for development of research in this area.

Key words: Environmental innovation; Optimization of products and processes; Process management; Sustainability. 


\section{INTRODUÇÃO}

O desenvolvimento de negócios sustentáveis tem recebido grande atenção nas últimas décadas. $\mathrm{O}$ surgimento de uma ordem de mudança econômica está levando empresas de todo o mundo a reverem padrões de produção e incluírem práticas de sustentabilidade nos serviços e produtos que oferecem (Ageron et. al., 2012). Como estas práticas sustentáveis não encontram espaço nos padrões de consumo e sistemas de produção tradicionais (Tseng et. al., 2012) é necessário que se adote novas culturas de consumo e novos modelos gerenciais e produtivos que vão ao encontro do conceito de sustentabilidade. Para alcançar este objetivo, a academia e o mercado têm se interessado pelo conceito de inovação ambiental, ou environmental innovation (Schiederig e Tietze, 2012).

Inovação ambiental inclui todos os tipos de inovações organizacionais que geram benefícios para o meio ambiente, abrangendo as mudanças e novidades organizacionais que buscam reduzir seus impactos ambientais (Kammerer, 2009). Neste contexto, inovação ambiental é a chave para a sustentabilidade, tanto em termos econômicos quanto ecológicos (Bernauer et. al., 2006). A inclusão das questões de inovação ambiental nos negócios, porém, exige muitas mudanças que devem ser feitas nos processos, produtos e serviços que levem a sensibilização para a responsabilidade ambiental (Tseng et. al., 2012). A gestão por processo, ou BPM, é um modelo gerencial que pode auxiliar as organizações a passarem por essas mudanças, corroborando com a implementação da inovação ambiental. Assim como na gestão por processos, Zhu e Sarkis (2004) verificaram que a obtenção de um compromisso dos gestores tem uma influência significativa sobre o sucesso da implementação da inovação ambiental.

A fim de fazer um paralelo entre os conceitos, a proposição deste artigo é evidenciar que a gestão por processos, com seu foco no entendimento, na modelagem, melhoria e otimização de processos de negócios pode auxiliar a inovação ambiental. Para encontrar evidências que colaborem com esta proposição, e em vista da crescente importância e aparente ligação dos temas, este trabalho tem o objetivo de fazer uma revisão sistemática da literatura entre os artigos que correlacionam os assuntos.

Esta pesquisa está dividida em cinco seções, sendo a primeira esta introdução; a segunda, contendo a fundamentação teórica do artigo; a terceira, o método adotado na revisão sistemática; e a quarta, os resultados obtidos. Por fim, a última seção, as considerações finais.

\section{FUNDAMENTAÇÃO TEÓRICA}

Esta seção tem por objetivo delinear as principais características de inovação ambiental e gestão por processos (BPM).

\subsection{Inovação ambiental}

Uma elucidação sobre inovação ambiental foi necessária para desenvolver este artigo, visto a diversidade dos termos usados pelos pesquisadores relacionadas a esse tema (Schiederig e Tietze, 2012). Para isto, esta pesquisa usou a obra de Angelo et. al. (2012) que faz análise dos termos usados para inovação ambiental. Segundo este estudo, a literatura destaca três principais termos relacionados à inovação ambiental: inovação ambiental, green innovation, ou inovação verde, e eco-innovation, ou ecoinovação (Angelo et. al., 2012). A seguir, são apresentadas as definições de cada termo.

Kammerer (2009) define inovação ambiental como as inovações que tem um efeito benéfico sobre o ambiente natural, independentemente se este foi o principal objetivo da inovação. Inovação ambiental, geralmente, têm um efeito de transbordamento para os esforços de P\&D e produzem externalidades positivas, que melhoram a qualidade ambiental das organizações (Rennings, 2000).

O termo ecoinovação foi proposto em 1990 por dois pesquisadores suíços, Schaltegger e Sturm (Côté et al., 2006). Nas últimas décadas, a ecoinovação tem sido considerada como a 
produção, assimilação e exploração de um produto, serviço, processo, gestão de negócios ou novo método para a organização que resulta, ao longo de seu ciclo de vida, na redução de risco ambiental, poluição e outros impactos negativos do uso de recursos em comparação às alternativas relevantes (Arundel e Kemp, 2009).

Chen et al. (2006) definem a inovação verde como a inovação que está relacionada à produtos verdes ou processos verdes, incluindo a inovação em tecnologias que estão envolvidas na economia de energia, prevenção da poluição, reciclagem de resíduos, projetos de produtos verdes ou gestão ambiental das empresas. Inovações verdes são classificadas em tecnologia, gestão de funções, design de produto e aspectos do processo de produção (Tseng et. al., 2012b). O processo de inovação verde é usado como um instrumento para melhorar o processo de gestão ambiental das empresas (Chen et al., 2006).

Apesar dos diferentes termos, o conceito de inovação ambiental pode ser considerado o mesmo (Angelo et.al, 2012). Neste trabalho, foi escolhido usar o termo inovação ambiental, pois é o mais predominante na academia, segundo a pesquisa realizada por Angelo et. al. (2012).

\subsection{Gestão por processos}

Gestão por processos, ou BPM, não é um conceito inteiramente novo. Durante 1970, os métodos para trabalhar com processos foram desenvolvidos sob os rótulos de just-in-time e produção enxuta por Schonberger (1986). Nos anos 1980, o Sistema Toyota de Produção lançou um novo paradigma de desempenho ao mundo. Entre as características deste novo modelo de operação, se encontrava a melhoria sistemática dos processos.

Nas décadas de 1980 e 1990, o escopo do controle de processo foi ampliado para abranger a ênfase corporativa do período, incluindo todas as funções de uma organização. Neste período, uma grande parte da atenção foi voltada para a reengenharia de processos empresariais (Hammer e Champy, 1993).

A reengenharia de processos foi sinônima do renascimento do interesse na gestão de processos (Hammer e Champy, 1993). No entanto, as consequências de muitas incursões deste modelo ocasionaram cortes de pessoal e estouros de orçamento. Depois disso, Davenport (1993), com uma visão mais moderada, sugeriu uma abordagem menos agressiva que o modelo de reengenharia e passou a adotar a melhoria contínua dos processos de negócio e inovação. Ao longo dos dez anos seguintes, outras práticas de gestão, tais como gestão da qualidade total (TQM), os modelos de negócio excelência, normas como a ISO 9000, tomaram um foco crescente em processos (Stalk et. al., 1992).

Contemporaneamente, gestão por processos, ou BPM, é uma disciplina de gestão que exige das organizações uma mudança para o pensamento centrado nos processos e que ao longo dos últimos 15 anos tem recebido atenção crescente (Doebeli et. al., 2011).

A Gestão por Processos pode ser entendida como todos os esforços de uma organização em analisar e, continuamente, melhorar suas atividades fundamentais (Trkman, 2010). Ela inclui métodos, técnicas e ferramentas para apoiar o projeto, a criação, a gestão, e a análise de processos de negócios operacionais (Aalst et. al., 2003). Gestão por processos investiga o que a empresa faz e, a partir disso, administra ciclos de vida de melhorias e otimizações (Smith e Fingar, 2003).

A prática gerencial de gestão por processos, de acordo com a ABPMP (2009), pode ser caracterizada como um ciclo de vida contínuo de atividades integradas de gestão por processos que, pode ser sumarizada por um conjunto gradual e interativo de atividades que incluem: (1) Planejamento e estratégia; (2) Análise; (3) Desenho e Modelagem; (4) Implantação; (5) Monitoramento e Controle; e (6) Refinamento. Essas atividades são detalhadas na figura 1. A importância do ciclo de vida de gestão por processos ganha reforço em Ko et. al. (2009) ao afirmarem que, para entender as terminologias e os recursos de gestão por processos, deve-se começar com uma apreciação do ciclo de vida de gestão por processos. 


\begin{tabular}{|c|c|}
\hline Atividade & Descrição \\
\hline $\begin{array}{c}\text { Planejamento e } \\
\text { estratégia }\end{array}$ & $\begin{array}{c}\text { Envolve o desenvolvimento de um plano e uma estratégia dirigida a processos para } \\
\text { a organização. Essa fase estabelece a estratégia e o direcionamento do processo de } \\
\text { gestão por processos. }\end{array}$ \\
\hline Análise & $\begin{array}{c}\text { Incorpora várias metodologias com a finalidade de entender os atuais processos } \\
\text { organizacionais no contexto das metas e objetivos desejados. }\end{array}$ \\
\hline Modelagem & $\begin{array}{c}\text { Objetiva entender o processo e avaliar os fatores ambientais que habilitam ou } \\
\text { restringem o processo. }\end{array}$ \\
\hline Desenho design & $\begin{array}{c}\text { Envolve a criação de especificações para processos de negócio novos ou } \\
\text { modificados dentro de diversos contextos de processos. }\end{array}$ \\
\hline Implantação & $\begin{array}{c}\text { Implementa o resultado da análise iterativa e o ciclo de desenho. Trata desa de } \\
\text { gerenciamento de mudança organizacional e está orientado à melhoria contínua e } \\
\text { otimização de processo. }\end{array}$ \\
\hline $\begin{array}{c}\text { Monitoramento e } \\
\text { controle }\end{array}$ & $\begin{array}{c}\text { Fornecem a informação necessária para que gestores de processo ajustem recursos } \\
\text { a fim de atingir objetivos dos processos. }\end{array}$ \\
\hline
\end{tabular}

Figura 1- Atividades do ciclo de vida da gestão por processos

Fonte: elaborado pelos autores a partir da ABPMP (2009)

Definidas as principais características de inovação ambiental e gestão por processos, a seção seguinte tem o objetivo de apresentar o método usado neste trabalho.

\section{MÉTODO DE PESQUISA}

Esta seção tem por objetivo delinear o método do artigo. Como suporte para a elaboração deste trabalho, utilizou-se a obra de Godinho et. al. (2009) e Angelo et. al. (2012).

Foi realizada uma análise bibliométrica para entender a relação entre a gestão por processos e inovação ambiental. O primeiro passo da pesquisa foi a seleção de artigos sobre a temática gestão por processos (BPM) e inovação ambiental. Para uma busca mais abrangente do tema, foram utilizados os três principais termos referentes à inovação ambiental. A consulta dos artigos foi feita na base de dados Scopus. O período de busca selecionado foi de 2006 a 2012.

Os strings utilizados para filtrar as informações foram: "Business Process Management" AND "environmental innovation" OR "BPM" AND "environmental innovation" OR "BPM" AND "green innovation" OR "business process management" AND "green innovation" OR "Green Process Improvement" OR "Green BPM" OR "Sustainability" AND "business process management" OR "Sustainability" AND "BPM" OR "Business Process Management" AND "ecoinnovation" OR "BPM" AND "eco-innovation".

Os termos poderiam estar contidos nos títulos, nas palavras-chave ou nos resumos. A fim de manter o escopo e foco do trabalho foi necessário selecionar as áreas de pesquisa para alcançar o objetivo principal da pesquisa. Na busca realizada na base da Scopus, foram selecionadas apenas as áreas de: Business, Management and Accounting e Social Sciences. O idioma selecionado foi o inglês.

Após a busca dos artigos, foi feita a seleção dos trabalhos que seriam incluídos nesta pesquisa. O método de análise foi a revisão sistemática e, em seguida, foi realizado um resumo dos artigos, uma discussão e classificação destes.

\section{RESULTADOS}

Nesta seção, serão apresentados os resultados da pesquisa. Este estudo é dividido em três partes teóricas. Primeiramente, foi elaborado um quadro resumo da revisão sistemática contendo todos os artigos selecionados para o trabalho (Seção 4.1). Em seguida, foram elencados os principais pontos de cada trabalho sobre a relação entre inovação ambiental e gestão por processos (Seção 4.2). Por fim, foi realizada uma análise geral dos objetivos dos trabalhos e da relação entre eles (Seção 4.3). 


\subsection{Revisão sistemática}

Após a busca dos artigos usando os filtros descritos no método, foram encontrados 19 artigos e a partir disto foi realizada a leitura dos títulos e resumos dos trabalhos para selecionar os que seriam relevantes a esta pesquisa. Foram selecionados 14 trabalhos com base nos critérios adotados de concordância com o objetivo da pesquisa: os trabalhos precisavam, concomitantemente, abordar inovação ambiental e gestão por processos. A partir dos 14 artigos selecionados, foi realizado um quadro resumo da revisão sistemática conforme ilustra a figura 2.

\begin{tabular}{|c|c|c|c|c|}
\hline Autor & Título & Contribuição para o Estado da Arte & Método & $\begin{array}{l}\text { Instituição } \\
\text { de Origem }\end{array}$ \\
\hline $\begin{array}{l}\text { Tseng, } \\
\text { M.L., Chiu, } \\
\text { S.F., Tan, } \\
\text { R.R., Siriban- } \\
\text { Manalang, } \\
\text { A.B. (2012a) }\end{array}$ & $\begin{array}{l}\text { Sustainable consumption and } \\
\text { production for Asia: } \\
\text { sustainability } \\
\text { through green design and } \\
\text { practice }\end{array}$ & $\begin{array}{l}\text { Este artigo identificou que o avanço da } \\
\text { tecnologia verde, do consumo verde, da } \\
\text { inovação verde, dos modelos } \\
\text { apropriados de negócios sustentáveis e } \\
\text { da cadeia de suprimentos verdes são os } \\
\text { integrantes chaves para a promoção em } \\
\text { larga escala de consumo e da produção } \\
\text { sustentáveis. } \\
\end{array}$ & Empírico & $\begin{array}{l}\text { Lunghwa } \\
\text { University of } \\
\text { Science and } \\
\text { Technology, } \\
\text { Taiwan et. } \\
\text { Al. }\end{array}$ \\
\hline $\begin{array}{l}\text { Tseng, M.- } \\
\text { L., Wang, } \\
\text { R., Chiu, } \\
\text { A.S.F, Geng, } \\
\text { Y., Lin, Y.H. } \\
\quad(2012 b)\end{array}$ & $\begin{array}{c}\text { Improving performance } \\
\text { of green innovation practices } \\
\text { under uncertainty }\end{array}$ & $\begin{array}{l}\text { Este estudo apresentou evidências de } \\
\text { que, a partir do estudo de caso } \\
\text { apresentado, práticas de inovação verde } \\
\text { têm implicações para as operações da } \\
\text { pesquisa e da prática em gestão. }\end{array}$ & Empírico & $\begin{array}{l}\text { Lunghwa } \\
\text { University of } \\
\text { Science and } \\
\text { Technology, } \\
\text { Taiwan et. al. }\end{array}$ \\
\hline $\begin{array}{l}\text { Paunescu } \\
\text { C., Acatrinei, } \\
\text { C. }(2012)\end{array}$ & $\begin{array}{l}\text { Managing maturity } \\
\text { in process-based improvement } \\
\text { organizations: A perspective } \\
\text { of the Romanian companies }\end{array}$ & $\begin{array}{l}\text { Este artigo apresentou um modelo que } \\
\text { ajuda as organizações, interessadas em } \\
\text { manterem-se competitivas e } \\
\text { promoverem práticas sustentáveis, a } \\
\text { monitorarem e avaliarem o desempenho } \\
\text { dos seus processos. } \\
\end{array}$ & Empírico & $\begin{array}{l}\text { Bucharest } \\
\text { University of } \\
\text { Economic } \\
\text { Studies, } \\
\text { Bucharest, } \\
\text { Romania } \\
\end{array}$ \\
\hline $\begin{array}{l}\text { Lee, K.- } \\
\text { H., Kim, J.-W. } \\
\quad(2012)\end{array}$ & $\begin{array}{c}\text { Green new product } \\
\text { development and supplier } \\
\text { involvement: Strategic } \\
\text { partnership for green } \\
\text { innovation }\end{array}$ & $\begin{array}{l}\text { Este estudo identificou que os fatores } \\
\text { críticos de sucesso para DNP verdes } \\
\text { incluem tecnologia, gestão de fatores, } \\
\text { cooperativismo e poder de equilíbrio } \\
\text { para as relações de colaboração. }\end{array}$ & Empírico & $\begin{array}{c}\text { Griffith } \\
\text { Business } \\
\text { School, South } \\
\text { Korea }\end{array}$ \\
\hline $\begin{array}{l}\text { Chang, C.-H. } \\
\text { (2011b) }\end{array}$ & $\begin{array}{l}\text { The Influence of Corporate } \\
\text { Environmental Ethics on } \\
\text { Competitive Advantage: The } \\
\text { Mediation Role of Green } \\
\text { Innovation }\end{array}$ & $\begin{array}{l}\text { Este estudo trouxe evidências de que a } \\
\text { inovação de produtos verdes medeia à } \\
\text { relação positiva entre ética ambiental } \\
\text { corporativa e vantagem competitiva, } \\
\text { mas a inovação verde do processo não } \\
\text { tem este mesmo papel. }\end{array}$ & Empírico & $\begin{array}{l}\text { Tamkang } \\
\text { University, } \\
\text { Taiwan }\end{array}$ \\
\hline $\begin{array}{l}\text { Chang, C.-H. } \\
\text { (2011a) }\end{array}$ & $\begin{array}{c}\text { Green } \\
\text { innovation performance: } \\
\text { Antecedent and consequence }\end{array}$ & $\begin{array}{c}\text { Este estudo usou modelagem de } \\
\text { equações estruturais para explorar o } \\
\text { efeito positivo da ética ambiental } \\
\text { corporativa na vantagem competitiva } \\
\text { nas indústrias de manufaturas de Taiwan } \\
\text { por meio do medidor de desempenho de } \\
\text { inovação verde. }\end{array}$ & Empírico & $\begin{array}{l}\text { Tamkang } \\
\text { University, } \\
\text { Taiwan }\end{array}$ \\
\hline $\begin{array}{l}\text { Guoyou, } \\
\text { Q., Saixing, } \\
\text { Z., Chiming, } \\
\text { T., Haitao, } \\
\text { Y., Hailiang, } \\
\text { Z. (2011) }\end{array}$ & $\begin{array}{c}\text { Stakeholders' Influences on } \\
\text { Corporate Green } \\
\text { Innovation Strategy: A Case } \\
\text { Study of Manufacturing Firms } \\
\text { in China }\end{array}$ & $\begin{array}{c}\text { Os resultados empíricos da pesquisa } \\
\text { mostraram que os stakeholders } \\
\text { desempenham um papel importante na } \\
\text { condução das organizações ao adotarem } \\
\text { a estratégia inovação verde nos } \\
\text { processos e produtos. }\end{array}$ & Empírico & $\begin{array}{l}\text { University } \\
\text { Shanghai, } \\
\text { China et. al. }\end{array}$ \\
\hline $\begin{array}{l}\text { Houy, } \\
\text { C., Reiter, }\end{array}$ & $\begin{array}{c}\text { Towards Green BPM - } \\
\text { Sustainability and resource } \\
\end{array}$ & $\begin{array}{l}\text { Argumenta-se, por meio deste artigo, } \\
\text { que a aplicação de abordagens do campo }\end{array}$ & Teórico & $\begin{array}{l}\text { Institute for } \\
\text { Information }\end{array}$ \\
\hline
\end{tabular}

Revista de Gestão Social e Ambiental - RGSA, São Paulo, v. 7, n. 2, p. 37-52, maio/ago. 2013. 


\begin{tabular}{|c|c|c|c|c|}
\hline $\begin{array}{l}\text { M., Fettke, } \\
\text { P., Loos, P. } \\
\quad(2011)\end{array}$ & $\begin{array}{l}\text { efficiency through business } \\
\text { process management }\end{array}$ & $\begin{array}{l}\text { de gestão por processos podem apoiar } \\
\text { iniciativas de TI verde. }\end{array}$ & & $\begin{array}{l}\text { Systems, } \\
\text { Germany et. } \\
\text { al. }\end{array}$ \\
\hline $\begin{array}{l}\text { Chapple, } \\
\text { K., Kroll, } \\
\text { C., William } \\
\text { Lester, } \\
\text { T., Montero, } \\
\text { S. (2011) }\end{array}$ & $\begin{array}{l}\text { Innovation in } \\
\text { the green economy: An } \\
\text { extension of the } \\
\text { regional innovation system } \\
\text { model? }\end{array}$ & $\begin{array}{l}\text { Argumenta-se, por meio deste artigo, } \\
\text { que além da inovação verde, as } \\
\text { empresas emergentes necessitam de } \\
\text { ferramentas adicionais de apoio de redes } \\
\text { locais a fim de transformarem novas } \\
\text { ideias e produtos. }\end{array}$ & Empírico & $\begin{array}{l}\text { University of } \\
\text { California- } \\
\text { United States } \\
\quad \text { et. al. }\end{array}$ \\
\hline $\begin{array}{l}\text { Cleven, A., } \\
\text { Winter, } \\
\text { R., Wortmann, } \\
\text { F (2011) }\end{array}$ & $\begin{array}{c}\text { Process performance manage } \\
\text { ment as a basic concept for } \\
\text { sustainable business process } \\
\text { management - Empirical } \\
\text { investigation and research } \\
\text { agenda }\end{array}$ & $\begin{array}{l}\text { Este trabalho avançou na compreensão } \\
\text { da sustentabilidade econômica no } \\
\text { contexto de gestão por processos, } \\
\text { argumentando que Process Management } \\
\text { Performance (PPM) representa uma } \\
\text { abordagem básica para estabelecer e } \\
\text { manter a sustentabilidade econômica em } \\
\text { gestão por processos. }\end{array}$ & Empírico & $\begin{array}{l}\text { University of } \\
\text { St. Gallen, } \\
\text { China }\end{array}$ \\
\hline $\begin{array}{l}\text { Hailemariam, } \\
\text { G., Vom } \\
\text { Brocke, J. } \\
(2011)\end{array}$ & $\begin{array}{l}\text { What } \\
\text { is sustainability in business } \\
\text { process management? A } \\
\text { theoretical framework and its } \\
\text { application in the public } \\
\text { sector of Ethiopia }\end{array}$ & $\begin{array}{l}\text { Propõe-se, por meio deste estudo, a } \\
\text { análise do conceito de sucesso de gestão } \\
\text { por processo, argumentando que as } \\
\text { iniciativas desta natureza precisam levar } \\
\text { em conta a perspectiva das múltiplas } \\
\text { partes interessadas, a fim de serem bem- } \\
\text { sucedidas. }\end{array}$ & Empírico & $\begin{array}{l}\text { University of } \\
\text { Liechtenstein, } \\
\text { Liechtenstein }\end{array}$ \\
\hline $\begin{array}{l}\text { Ghose, } \\
\text { A., Hoesch- } \\
\text { Klohe, } \\
\text { K., Hinsche, } \\
\text { L., Le, L.-S. } \\
\text { (2010) }\end{array}$ & $\begin{array}{c}\text { Green business process } \\
\text { management: A research } \\
\text { agenda }\end{array}$ & $\begin{array}{l}\text { Este artigo descreve os contornos da } \\
\text { paisagem de pesquisa emergente em } \\
\text { gestão por processos verde e apresenta } \\
\text { alguns resultados iniciais nesta área. }\end{array}$ & Teórico & $\begin{array}{l}\text { University of } \\
\text { Wollongong, } \\
\text { Australia }\end{array}$ \\
\hline $\begin{array}{l}\text { Kralj, D. } \\
(2009)\end{array}$ & $\begin{array}{l}\text { Sytems thinking integrated } \\
\text { system approach to } \\
\text { sustainable management }\end{array}$ & $\begin{array}{l}\text { Este trabalho apresenta o pensamento } \\
\text { sistêmico como essencial para a } \\
\text { inovação de processos, sendo o } \\
\text { responsável pelo desenvolvimento de } \\
\text { uma cultura de gestão de inovação. }\end{array}$ & Teórico & $\begin{array}{l}\text { University of } \\
\text { Primorska, } \\
\text { Slovenia }\end{array}$ \\
\hline $\begin{array}{l}\text { Chen, Y.- } \\
\text { S., Lai, S.- } \\
\text { B., Wen, C.-T. } \\
\quad \text { (2006) }\end{array}$ & $\begin{array}{c}\text { The influence of green } \\
\text { innovation performance on } \\
\text { corporate advantage in } \\
\text { Taiwan }\end{array}$ & $\begin{array}{l}\text { Este estudo identifica as performances } \\
\text { de inovação de produtos verdes e } \\
\text { inovação verde dos processos que estão } \\
\text { positivamente correlacionadas com a } \\
\text { vantagem corporativa competitiva. }\end{array}$ & Empírico & $\begin{array}{l}\text { National } \\
\text { Yunlin } \\
\text { University of } \\
\text { Science and } \\
\text { Technology, } \\
\text { Taiwan et. al. }\end{array}$ \\
\hline
\end{tabular}

Figura 2 - Revisão sistemática

Fonte: Autores

\subsection{Análise dos artigos}

Após a construção Figura 2, foram elaboradas análises resumos de cada trabalho, conforme segue, destacando seus pontos principais e os paradigmas encontrados sobre inovação ambiental e gestão por processos.

Os autores Paunescu e Acatrinei (2012) destacam que, cada vez mais, as organizações são responsáveis por mostrarem atitudes, processos e produtos mais ambientalmente conscientes aos seus clientes internos e externos (Păunescu e Acatrinei , 2012). No trabalho "Managing maturity in process-based improvement organizations: A perspective of the Romanian companies" fica destacado que uma empresa bem-sucedida deve ter a capacidade de acompanhar e avaliar continuamente o ambiente externo para os desafios, bem como para analisar o seu ambiente interno de oportunidades de melhoria contínua. Além disso, para alcançar um desempenho superior de negócios, as empresas devem desenvolver um processo sistemático de monitoramento contínuo e controle de desempenho. Estes conceitos ficam evidentes quando uma organização decide promover 
a gestão por processos. Baseados em outros modelos da literatura, Păunescu e Acatrinei (2012) apresentam, no artigo, um modelo que ajuda as organizações a monitorarem e avaliarem o desempenho dos processos e alcançarem níveis mais altos de sustentabilidade. Desta forma, acredita-se que por meio da gestão por processos tornar-se-ia mais fácil avaliar o desempenho dos processos, e consequentemente, promover níveis mais altos de sustentabilidade. Os resultados da pesquisa evidenciam que existem fortes correlações positivas entre as variáveis analisadas, entre elas, processos e inovação ambiental.

Os autores Houy et. al. (2011) destacam que no debate sobre a sustentabilidade, um dos tópicos centrais é a eficiência energética das infraestruturas de tecnologia da informação. Iniciativas em Tecnologia da Informação (TI) verde incluem investimentos para a redução do consumo de energia, virtualização de servidores, métodos de organização, treinamento de pessoal, redução de resíduos, entre outros, estando diretamente relacionados com a gestão por processos (BPM) (Houy et. al., 2011). Os autores elucidam que técnicas e ferramentas de gestão por processo têm de ser adaptadas às exigências da TI verde. As técnicas e ferramentas resultantes dessa adaptação são resumidas sob o termo "Green BPM" (Houy et. al., 2011). Argumenta-se, portanto, que, nesse contexto, gestão por processos pode fundamentalmente apoiar iniciativas de TI verde e, assim, melhorar a sustentabilidade e a eficiência dos recursos das atividades empresariais em geral. A gestão por processos pode ser diretamente relacionada à TI, uma vez que ao se pensar em automação de processos, tem-se como premissa trabalhar com processos. Por meio da gestão por processos são oferecidas técnicas adequadas para o controle de execução de design, bem como a análise de processos de negócios, a fim de melhorar a eficácia e eficiência de criação de valor na organização (Houy et. al., 2011). Por fim, os autores elucidam que a discussão sobre a sustentabilidade por meio da "green BPM" ainda está em seus estágios iniciais e que apenas técnicas rudimentares existem até ao momento. Isto evidencia o argumento de que a gestão por processos corrobora com a inovação ambiental, porém poucos estudos ainda abordam os temas e que, talvez, o termo "green BPM" possa ser melhor desenvolvido para não apenas abordar a TI, mas os processos não automatizados também.

Os autores Cleven et. al. (2011) iniciam seu trabalho dizendo que desenvolvimento sustentável, organização sustentável e estratégias de sustentabilidade são termos intensamente discutidos na comunidade de negócios contemporânea. No entanto, o conceito de sustentabilidade ainda permanece vago, especialmente quanto ao seu significado e suas implicações para o campo da gestão por processos. Mais uma vez, evidencia-se que os conceitos gestão por processos e inovação ambiental são correlatos, porém os trabalhos acadêmicos na área são escassos. Propõe-se, por meio deste artigo, portanto, avançar na compreensão da sustentabilidade econômica no contexto da gestão por processos. Argumenta-se que Process Management Performance (PPM) representa uma abordagem básica para estabelecer e manter a sustentabilidade econômica em gestão por processos. Embora a dimensão econômica da sustentabilidade tenha o maior nível de maturidade, uma investigação empírica revela que as organizações estão enfrentando grandes dificuldades com a sua implementação, em particular no nível dos processos. Isso mostra como estudos empíricos poderiam auxiliar os gestores a superar essas dificuldades na promoção dos processos.

$\mathrm{O}$ artigo "What is sustainability in business process management? A theoretical framework and its application in the public sector of Ethiopia", de Hailemariam e Vom Brocke (2011), inicia argumentado que mesmo havendo uma variedade de métodos para gestão por processos, iniciativas pontuais desta gestão ainda encontram barreiras para serem bem-sucedidas na prática. Tendo como base a teoria dos stakeholders e argumentando que as iniciativas de gestão por processos precisam considerar a perspectiva das múltiplas partes interessadas (gestores, acionistas, funcionários, etc), este trabalho faz uma avaliação do modelo proposto no caso de um grande projeto de gestão por processos no setor público etíope. $\mathrm{O}$ estudo de caso revela a ligação direta entre gestão por processos e inovação ambiental. Entre as melhorias propostas, a inovação ambiental aparece como um dos alvos a serem alcançados, porém a implementação destas melhorias ainda não foram 
avaliadas. Por fim, Hailemariam e Vom Brocke (2011) finalizam o artigo delineando oportunidades para pesquisas futuras.

O artigo de Ghosa et. al. (2010) argumenta que há consenso mundial sobre a necessidade de reduzir globalmente a emissão de carbono. Porém, enquanto a atenção da academia e do mercado tem sido centrada no desenvolvimento de fontes alternativas de energia, tecnologias automotivas ou técnicas de eliminação de resíduos, muitas vezes, ignora-se o fato de que a capacidade de otimizar operações para reduzir o impacto de emissões é fundamental para este exercício. Isto significa que, pensar nos processos antes da tecnologia pode contribuir com a inovação ambiental. Assim como no trabalho de Houy et. al. (2011), Ghosa et. al. (2010) usam o termo "green BPM" para descrever uma nova classe de tecnologias que aproveitam a gestão por processos para permitir que o processo de concepção de novas tecnologias, execução e monitoramento sejam alimentados com informações da pegada de carbono. O artigo descreve, então, os contornos da paisagem de pesquisa emergente em "green BPM" e apresenta alguns resultados iniciais nesta área de tecnológica da gestão por processos. O trabalho é finalizado concluindo que a tecnologia de modelagem de processo tem aplicações além do que tradicionalmente se descreve como processos de negócios, pois também podem modelar e melhorar a produção e outros processos "físicos" das organizações. Isto é, os processos de negócios e os processos de produção têm impacto na promoção da inovação ambiental, segundo Ghosa et. al. (2010).

O trabalho de Tseng et. al. (2012a) é iniciado afirmando que há uma necessidade urgente de desenvolver e aplicar adequadamente políticas que promovam o design verde, a cadeia de suprimentos verde, produção enxuta, proteção de saúde e segurança ao trabalhador e consumidor. Todos estes conceitos estão relacionados à gestão por processos. No caso deste artigo, portanto, a gestão por processos aparece como uma influência positiva indireta à promoção da inovação verde. Tseng et. al. (2012a) argumenta que as organizações podem embarcar no fornecimento de produtos e serviços verdes, construídos tendo como base expectativas tácitas e explícitas dos consumidores. Isso poderia ajudar a incentivar os produtores a tomar ações mais radicais de inovação verde, inclusive em seus processos. Os autores fazem uma lista das principais lições resultantes da mesa redonda sobre consumos e produção sustentáveis da Ásia-Pacífico: política como um abrangente propiciador da sustentabilidade; tecnologia; consumo verde; inovações verdes e modelo de negócios adequados e sustentáveis; gestão da cadeia de suprimento verde (Tseng et. al., 2012a). Estas iniciativas podem contribuir para o progresso da sociedade, mantendo os limites não negociáveis da capacidade de suporte do ecossistema (Tseng et. al., 2012a).

O estudo de Tseng et. al. (2012b) centrou-se no desenvolvimento de uma avaliação quantitativa de inovação verde sob incerteza em que quatro aspectos foram considerados e avaliados simultaneamente. $\mathrm{O}$ modelo proposto, com uma estrutura hierárquica e linguística, estabeleceu uma base para futuras pesquisas e pode ser usado para prever aspectos de incerteza (Tseng et. al., 2012b). Pode também ser aplicado para avaliar e determinar a inovação verde em práticas que vão ajudar a melhorar o desempenho ambiental das organizações. O artigo é finalizado concluindo que há evidências de que a sistematização de inovações de gestão será um fator crítico de sucesso para as empresas do século 21 (Tseng et. al., 2012b). Esta sistematização de inovações de gestão pode ser melhor alcançada por meio da gestão por processos, isto é, esta abordagem aparece como um colaboração indireta da inovação ambiental neste trabalho (Tseng et. al., 2012b).

O trabalho de Lee e Kim (2012) inicia argumentando que integrar as preocupações ambientais com Desenvolvimento de Novos Produtos (DNP) tornou-se uma questão estratégica para a competitividade empresarial. Apesar da atenção crescente de produtos verdes na gestão da cadeia de abastecimento, existe uma lacuna emergente sobre DNP verdes na tradicional literatura sobre Desenvolvimento de Novos Produtos. Na tentativa de preencher esta lacuna, este estudo apresenta um caso em que fornecedores-chave coreanos conseguiram gerir os desafios ambientais e o desenvolvimento de uma geladeira verde por meio de uma relação de colaboração. Esta pesquisa é desenvolvida para uma melhor compreensão do processo DNP e dos fatores críticos que contribuíram para a produção de uma geladeira verde. A contribuição da gestão por processos na 
inovação neste trabalho também se dá de forma indireta, uma vez que para o DNP é preciso organizar e trabalhar sob a ótica dos processos.

O estudo de Chang (2011a) utiliza modelagem de equações estruturais (MEE) para explorar o efeito da ética ambiental em termos de vantagem competitiva na indústria de fabricação de Taiwan por meio do mediador: desempenho de inovação verde. Este artigo divide a inovação verde em inovação verde de produto e do processo. Os resultados empíricos mostram que a ética ambiental afeta positivamente ambos os fatores. Além disso, o estudo verifica que o desempenho da inovação verde de produtos medeia a relação positiva entre ética ambiental corporativa e vantagem competitiva, mas já a inovação verde do processo não o faz. Este resultado é diferente dos outros trabalhos. Para Chang (2011a), a inovação verde dos processos não estaria relacionada à ética ambiental corporativa.

O segundo trabalho de Chang (2011b) é uma continuação de "Green innovation performance: Antecedent and consequence". Neste estudo, utiliza-se a modelagem de equações estruturais (MEE) para explorar o efeito positivo corporativo da ética ambiental na vantagem competitiva nas indústrias de fabricação de Taiwan por meio do mediador: desempenho de inovação verde. Segundo os resultados da pesquisa, ética ambiental coorporativa não só pode afetar diretamente a vantagem competitiva, mas também influenciá-la indiretamente por intermédio de inovação verde de produtos na indústria de fabricação de Taiwan (Chang, 2011b).

Para enfrentar o desafio da degradação ambiental na China, um número crescente de empresas começou a integrar sistemas de gestão ambiental em suas estratégias de negócios e desenvolver estratégias de inovação verde (Guoyou et. al., 2011). Com base na teoria dos stakeholders, o artigo "Stakeholders' Influences on Corporate Green Innovation Strategy: A Case Study of Manufacturing Firms in China" tenta explorar as influências das partes interessadas sobre a implementação de inovação verde do processo e a inovação verde de produtos. Os resultados empíricos mostram que os clientes estrangeiros desempenham um papel importante na condução de empresas ao adotarem a estratégia de inovação verde do processo. Tal fato reforça a hipótese de que o comércio internacional aumenta a difusão da inovação de práticas verdes, que são consistentes com as descobertas anteriores de que o comércio internacional desempenha um papel importante na difusão de práticas ambientais eficientes (Guoyou et. al., 2011). Apesar deste trabalho estar focado no papel dos stakeholders, evidencia-se a importância de um processo verde, e que este processo tem como resultado a difusão das práticas ambientais. Sendo assim, existe neste artigo a evidência de uma correlação positiva direta entre gestão por processos e inovação ambiental.

O objetivo do estudo de Chen et. al. (2006) é identificar se o desempenho da inovação verde trouxe efeito positivo para a vantagem competitiva. A pesquisa constatou que as performances de inovação verde do processo e de produtos estão positivamente correlacionadas com a vantagem competitiva. $\mathrm{O}$ estudo salientou, porém, que as empresas devem conhecer o valor correto e o posicionamento da inovação verde antes de investirem.

O trabalho de Chapple et. al. (2011) argumenta que os formuladores de políticas públicas têm tido uma preocupação cada vez maior com a inovação verde como fonte de criação de emprego. Usando o caso da Califórnia, argumenta-se que a inovação verde modifica modelos tradicionais de inovação e o próprio desenvolvimento econômico. O estudo de Chapple et. al. (2011) utiliza dados para definir economia verde, identificar a inovação de produtos e serviços e apontar o crescimento setorial e regional por meio da inovação verde. As organizações mais ambientalmente desafiadas estão entre as mais propensas a criar novos processos. Ao pensar em novos processos, é possível concluir que organizações que já promovem a gestão por processos têm uma vantagem em relação àquelas baseadas na gestão funcional, evidenciando o papel deste tipo de gestão na inovação ambiental estudada pelos autores.

Kralj (2009) inicia seu artigo afirmando que uma das principais razões dos problemas organizacionais contemporâneos é a ausência de uma abordagem integral no planejamento ou na elaboração de estratégias de desenvolvimento. Isto é, ausência da promoção da gestão por processos. A teoria sobre a base da experiência prática de planejamento prevê o desenvolvimento 
sustentável como um processo contínuo de desenvolvimento bem-sucedido de melhoramento. Processos contínuos de melhoramento podem ser melhor alcançados em organizações que já promovem a gestão por processos. A promoção de inovação verde em um sistema organizacional pode produzir uma economia significativa na quantidade de recursos e resultar, portanto, em um menor impacto ambiental. Kralj (2009) argumenta que a abordagem de um sistema integrado conecta as exigências do desenvolvimento sustentável à excelência ambiental com os requisitos de negócios.

\section{ANÁLISE E DISCUSSÃO}

Esta seção tem por objetivo delinear as características gerais dos artigos abordadas na seção anterior. Apresenta-se uma figura conceitual dos principais aspectos que relacionam a gestão por processos com inovação ambiental. Por fim, é realizada uma classificação dos trabalhos da revisão sistemática, a fim de ilustrar, de forma sintética, as contribuições dos artigos e a relação entre eles.

Esta pesquisa teve como objetivo realizar uma varredura nos artigos que tratam de inovação ambiental e que fazem relação do tema com gestão por processos. De uma forma geral, a gestão por processos é apresentada como auxiliar, direta ou indireta, nas iniciativas de inovação ambiental, como destaca o trabalho de Houy et. al. (2011). Não houve trabalhos argumentando a favor da não relação dos temas.

A primeira consideração a ser feita é que há poucos artigos tratando da relação dos temas. Dentro do período selecionado para a pesquisa, foram encontrados apenas 14 estudos. O número reduzido de artigos evidencia a pouca exploração do assunto e a possível necessidade de maior produção científica nesta área, tendo em vista a relevância deste tema. No filtro utilizado na busca dos artigos, foi incluído o período de 2006 a 2012, e onze dos artigos foram publicados entre 2011 e 2012, sendo três deles ainda in press, o que corrobora com a evidência de contemporaneidade do tema e a sua tendência de novas publicações. Um dos próprios autores salienta que o conceito de sustentabilidade ainda permanece vago, especialmente o seu significado e implicações para o campo de gestão por processos (Cleven et. al., 2011).

Continuando a análise, alguns trabalhos evidenciam a falta de pesquisa empírica examinando a maturidade de processos em inovação ambiental, salienta-se, porém, que 11 trabalhos dos artigos aqui selecionados eram estudos empíricos e apenas 3 teóricos. Para corroborar com a busca dos artigos desta pesquisa, foi utilizada a obra de Angelo et. al. (2012) que auxiliou este estudo ao fazer o levantamento dos principais termos referentes à inovação ambiental, aumentando assim o escopo da busca. A maioria dos trabalhos desta pesquisa usou o termo inovação verde ao abordar o tema, diferente dos resultados apresentados por Angelo et. al. (2012) na sua busca por termos ligados à inovação ambiental. Isto pode ter ocorrido porque, apesar do conceito ser o mesmo, o termo inovação verde está diretamente relacionado à processos verdes e, portanto, à gestão por processos.

Pode-se considerar também que a gestão por processos é capaz de realizar o papel que os autores Păunescu e Acatrinei (2012) consideraram fundamentais para uma empresa ser bemsucedida: colaborar com a capacidade de acompanhar e avaliar continuamente o ambiente externo para os desafios, bem como para analisar o seu ambiente interno de oportunidades de melhoria contínua e inovação ambiental.

Para melhor identificar os pontos principais de contribuição da gestão por processos na inovação ambiental e identificar outros pontos que relacionam os temas, foi elaborado uma figura conceitual ilustrada na figura 3. 


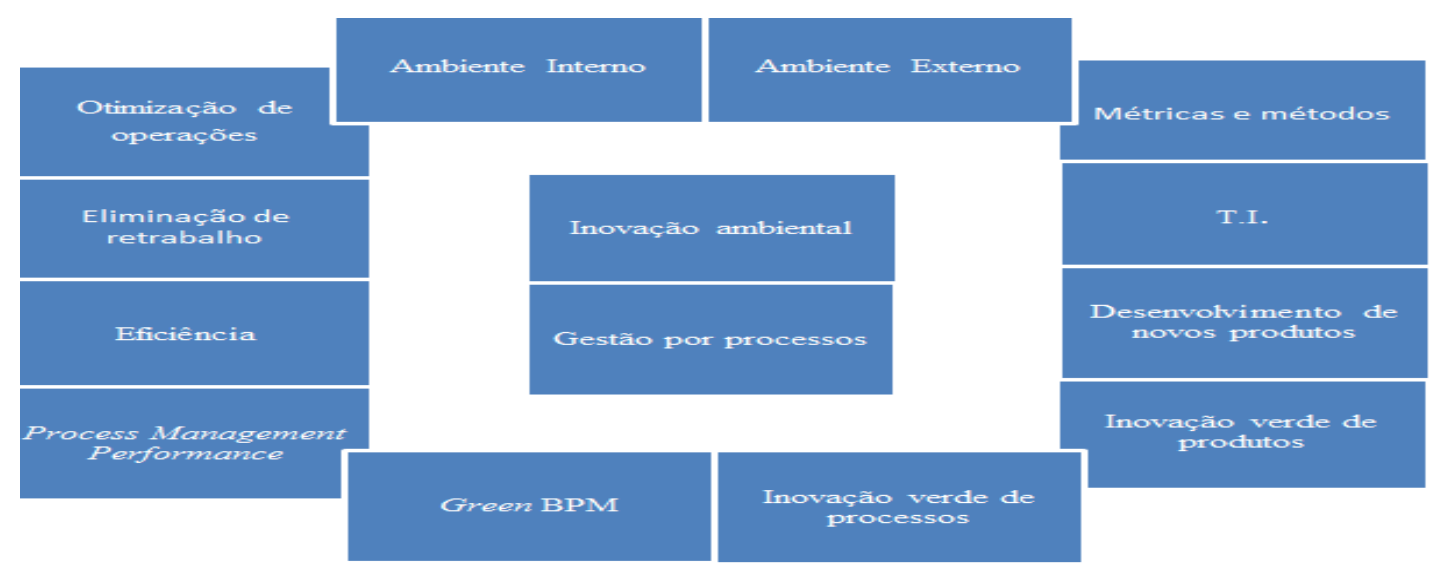

Figura 3 - Figura conceitual

Fonte: Autores

A figura conceitual é uma tentativa de reunir os principais aspectos encontrados nesta pesquisa que relacionam os temas estudados. Por isso, os termos inovação ambiental e gestão por processos estão no centro da figura, pois representam o foco da pesquisa, e ao redor, estão os principais pontos encontrados, a partir da pesquisa, que correlacionam os temas. Estas partes evidenciam que a gestão por processos contribui para o processo da inovação ambiental.

Por fim, após resumir os artigos, foi possível criar uma classificação entre os trabalhos para melhor entender a relação entre eles e quais foram suas colaborações para esta pesquisa. As categorias aqui encontradas foram:

a) Fatores e práticas que contribuem para a sustentabilidade.

b) Processos e sustentabilidade: a contribuição da gestão por processos para a sustentabilidade.

c) Avaliação dos fatores e práticas que contribuem para a sustentabilidade.

De uma forma geral, todos os artigos apresentados na revisão bibliográfica tratam das contribuições de gestão por processos para a sustentabilidade. Porém, os artigos classificados na categoria A incluem, além disto, outros fatores e práticas para a contribuição da sustentabilidade, como, por exemplo, Tecnologia de Informação, Corporativismo, Visão Sistêmica e etc. Esses artigos abordaram a gestão por processos de uma forma indireta na contribuição à inovação ambiental. As discussões realizadas nos trabalhos classificados na categoria $\mathrm{A}$ fazem alusão às melhorias promovidas pela gestão por processos como promotoras da inovação ambiental, mas não citavam a abordagem de gestão baseada em processos de forma direta. $\mathrm{O}$ resultado de cada trabalho pode ser analisado na seção anterior de discussão dos artigos. Conclui-se que estes artigos colaboram com a proposição desta pesquisa, pois, mesmo que de forma indireta, todas as contribuições da gestão por processos têm relações positivas com a inovação ambiental.

Os artigos classificados na categoria B tratam dos aspectos da gestão por processos na sustentabilidade. Estes artigos foram os mais evidentes em relação à contribuição da gestão por processos com a inovação ambiental. Termos com "green BPM" foram usados em dois artigos, mostrando a gestão por processos como uma premissa para a inovação ambiental. Isto é, a gestão por processos foi abordada, de forma direta e elementar, para contribuir com a gestão ambiental. Ainda na categoria $\mathrm{B}$, alguns artigos correlacionaram um processo bem desenhado e conhecido com o termo processo verde, que seria um resumo de um processo sem retrabalho, sem resíduos, conforme a gestão por processos tenta promover. Estes processos verdes seriam, portanto, importantes na promoção da inovação ambiental.

Já os artigos classificados na categoria C, que têm apenas dois trabalhos, fazem uma avaliação das práticas e dos fatores que contribuem com a sustentabilidade. O papel desempenhado pela gestão por processos, assim como na categoria A, é mostrado, de forma indireta, na promoção da inovação ambiental nestes artigos. Chang (2011a) e Chang (2011b), porém, contra argumentam que os chamados processos verdes não teriam um papel mediador entre a promoção da ética 
ambiental e a vantagem competitiva das organizações. Isto não é uma evidência contra o argumento deste trabalho, pois se salienta que os produtos verdes, produzidos a partir destes processos verdes, fariam o papel destes mediadores. Apesar destes dois estudos se mostrarem um pouco distantes da proposição deste trabalho, os artigos permanecem sobre a evidência das correlações positivas entre gestão por processos e inovação ambiental.

Na figura 4 é apresentada a distribuição dos artigos conforme a classificação criada nesta pesquisa.

\begin{tabular}{|c|c|c|}
\hline Autor & Título & Classificação \\
\hline $\begin{array}{l}\text { Tseng, M.L., Chiu, S.F., Tan, } \\
\text { R.R., Siriban-Manalang, A.B. } \\
\text { (2012a) }\end{array}$ & $\begin{array}{l}\text { Sustainable consumption and production for Asia: sustainability } \\
\text { through green design and practice }\end{array}$ & A \\
\hline $\begin{array}{l}\text { Tseng, M.-L., Wang, R., Chiu, } \\
\text { A.S.F, Geng, Y., Lin, Y.H. } \\
\text { (2012b) }\end{array}$ & $\begin{array}{l}\text { Improving performance of green innovation practices under } \\
\text { uncertainty }\end{array}$ & A \\
\hline $\begin{array}{l}\text { Paunescu, C., Acatrinei, C. } \\
\text { (2012) }\end{array}$ & $\begin{array}{c}\text { Managing maturity in process-based improvement organizations: A } \\
\text { perspective of the Romanian companies }\end{array}$ & B \\
\hline Lee, K.-H., Kim, J.-W. (2012) & $\begin{array}{c}\text { Green new product development and supplier involvement: Strategic } \\
\text { partnership for green innovation }\end{array}$ & A \\
\hline Chang, C.-H. (2011b) & $\begin{array}{c}\text { The Influence of Corporate Environmental Ethics on Competitive } \\
\text { Advantage: The Mediation Role of Green Innovation }\end{array}$ & $\mathrm{C}$ \\
\hline Chang, C.-H. (2011a) & Green innovation performance: Antecedent and consequence & $\mathrm{C}$ \\
\hline $\begin{array}{l}\text { Guoyou, Q., Saixing, } \\
\text { Z., Chiming, T., Haitao, } \\
\text { Y., Hailiang, Z. (2011) }\end{array}$ & $\begin{array}{l}\text { Stakeholders' Influences on Corporate Green Innovation Strategy: A } \\
\text { Case Study of Manufacturing Firms in China }\end{array}$ & A \\
\hline $\begin{array}{l}\text { Houy, C., Reiter, M., Fettke, } \\
\text { P., Loos, P. (2011) }\end{array}$ & $\begin{array}{c}\text { Towards Green BPM - Sustainability and resource efficiency } \\
\text { through business process management }\end{array}$ & B \\
\hline $\begin{array}{l}\text { Chapple, K., Kroll, } \\
\text { C., William Lester, } \\
\text { T., Montero, S. (2011) }\end{array}$ & $\begin{array}{l}\text { Innovation in the green economy: An extension of the } \\
\text { regional innovation system model? }\end{array}$ & A \\
\hline $\begin{array}{c}\text { Cleven, A., } \\
\text { Winter, R., } \\
\text { Wortmann, F (2011) }\end{array}$ & $\begin{array}{c}\text { Process performance management as a basic concept for } \\
\text { sustainable business process management - Empirical investigation } \\
\text { and research agenda }\end{array}$ & B \\
\hline $\begin{array}{l}\text { Hailemariam, G., Vom } \\
\text { Brocke, J. (2011) }\end{array}$ & $\begin{array}{l}\text { What is sustainability in business process management? A theoretical } \\
\text { framework and its application in the public sector of Ethiopia }\end{array}$ & B \\
\hline $\begin{array}{l}\text { Ghosa, A., Hoesch-Klohe, } \\
\text { K., Hinsche, L., Le, L.-S. } \\
\text { (2010) }\end{array}$ & Green business process management: A research agenda & B \\
\hline Kralj, D. (2009) & $\begin{array}{c}\text { Sytems thinking integrated system approach to sustainable } \\
\text { management }\end{array}$ & A \\
\hline $\begin{array}{l}\text { Chen, Y.-S. , Lai, S.-B., Wen, } \\
\text { C.-T. (2006) }\end{array}$ & $\begin{array}{l}\text { The influence of green innovation performance on corporate } \\
\text { advantage in Taiwan }\end{array}$ & B \\
\hline
\end{tabular}

Figura 4 - Classificação dos artigos

Fonte: Os autores

A análise do quadro e dos conteúdos dos artigos permite concluir que há evidências para acreditar ser verdade a proposição inicial artigo. É possível que gestão por processos, com seu foco no entendimento, na modelagem, melhoria e otimização de processos de negócios contribua com a inovação ambiental. Os trabalhos, de uma forma geral, evidenciam esta contribuição. Alguns autores afirmam que o desenvolvimento de práticas ambientais nas organizações surgem da otimização das operações para conduzir seu desenvolvimento ambiental e reduzir os impactos negativos. Esta otimização de operações pode ser alcançada com a gestão por processos, isso porque a gestão por processos compreende todos os esforços de uma organização em analisar e continuamente melhorar suas atividades fundamentais. 


\section{CONSIDERAÇÕES FINAIS}

Este estudo teve como objetivo realizar uma análise nos artigos que tratam de inovação ambiental e que fazem relação do tema com a gestão por processos. A partir dos 14 trabalhos encontrados nesta pesquisa, foi possível fazer algumas considerações. Primeiramente, a pesquisa evidencia que a gestão por processos é abordada em todos os artigos como uma prática colaborativa para a inovação ambiental, porém, parte dos trabalhos abordam outros fatores, além da gestão por processos, como contribuintes para a sustentabilidade. Apenas seis trabalhos abordaram, de forma clara e exclusiva, a relação da gestão por processos com a inovação ambiental.

Alguns autores afirmam que o desenvolvimento de práticas ambientais nas organizações precisa da otimização das operações para conduzir seu desenvolvimento ambiental e reduzir os impactos negativos. Esta otimização de operações pode ser alcançada com a gestão por processos.

Além disso, é possível verificar que muitos dos trabalhos estavam relacionados entre si. A partir da classificação sugerida neste artigo, foi possível identificar três nichos de assuntos abordados pelas pesquisas: Fatores e práticas que contribuem para a sustentabilidade; processos e sustentabilidade: a contribuição da gestão por processos para a sustentabilidade e, por fim, avaliação dos fatores e práticas que contribuem para a sustentabilidade.

O número reduzido de artigos evidencia a pouca exploração do assunto e a necessidade de maior produção científica nesta área. $\mathrm{O}$ fato dos artigos se concentrarem nas publicações dos anos 2011 e 2012 corrobora com a evidencia de contemporaneidade do tema e a sua tendência de novas publicações. Ainda salienta-se que o conceito de sustentabilidade permanece vago, especialmente o seu significado e suas implicações para o campo de gestão por processos.

Por fim, após identificar um marco teórico dos temas, este artigo buscou contribuir com uma discussão preliminar sobre o assunto. Acredita-se que é possível promover pesquisas na área desenvolvendo o termo "green BPM", usado em dois trabalhos em que os próprios autores argumentaram haver a necessidade de mais estudos. Acredita-se também haver a possibilidade de fazer estudos que comparem organizações que promovem inovação ambiental com base nas abordagens gestão por processos e organizações que promovem a inovação ambiental a partir da gestão funcional. Por fim, acredita-se haver a possibilidade de pesquisas que analisem o caminho inverso proposto por esse artigo, trabalhos que procurem evidências da colaboração da inovação verde entre as organizações que ainda vão promover a gestão por processos.

A originalidade principal deste estudo foi propor a revisão sistemática entre os conceitos de gestão por processos e inovação ambiental. Como qualquer estudo de natureza teórica, é necessário avançar com a integração desses conceitos aqui revisados com a práxis organizacional.

\section{REFERÊNCIAS}

Aalst, W., Hofstede, A., Weske, M. (2003) Business process management : a survey, BPM'03 Proceedings of the 2003 international conference on Business process management, 1-12.

Abpmp. (2009). Guia para o Gerenciamento de Processos de Negócio (BPM).

Ageron, B., Gunasekaran, A., Spalazani, A. (2012) Sustainable supply management: An empirical study. International Journal of Production Economics, 140, 168-182.

Angelo, F. D., Jabbour, C. J. C., Galina, S. V. (2012) Environmental innovation: in search of a meaning. World Journal of Entrepreneurship, Management and Sustainable Development, 8, 113 121.

Arundel, A. e Kemp, R. (2009) Measuring ecoinnovation. United Nations University - Maastricht Economic and Social Research and Training Centre on Innovation and Technology. 
Bernauer, T., Engels, E., Kammerer, D. e Seijas, J. (2006) Explaining green innovation: ten years after porter's win-win proposition: how to study the effects of regulation on corporate environmental innovation? Center for Comparative and International Studies.(17).

Chang, C.-H. (2011a) The influence of corporate environmental ethics on competitive advantage: the mediation role of green innovation. Journal of Business Ethics,104, 361-370.

Chang, C.-H. (2011b) Green innovation performance: antecedent and consequence. (2011b) PICMET: Portland International Center for Management of Engineering and Technology, Proceedings. Article number 6017872.

Chapple, K. Kroll, C., Lester, T., Montero, S. (2011) Innovation in the green economy: An extension of the regional innovation system model? Economic Development Quarterly, 25, 5-25.

Chen, Y.-S., Lai, S.-B., Wen, C.-T. (2006) The influence of green innovation performance on corporate advantage in Taiwan. Journal of Business Ethics, 67, 331-339.

Cleven, A., Winter, R., Wortmann, F. (2011) Process performance management as a basic concept for sustainable business process management - Empirical investigation and research agenda. Lecture Notes in Business Information Processing 8th International Workshops and Education Track on Business Process Management, 66, 479-488.

Côté, R., Booth, A., Louis, B. (2006) Eco-efficiency and SMEs in Nova Scotia, Canada". Journal of Cleaner Production, 14, 542-50.

Davenport, T.H. (1993) Process innovation. Harvard Business School Press. Boston, MA.

Doebeli,G., Fisher, R., Gapp, R., Sanzogni. (2011) Using BPM governance to align systems and practice. Business Process Management Journal,17, 184-202.

Ghose, A., Hoesch-Klohe, K., Hinsche, L., LE, L-S. (2010) Green business process management: a research agenda. Australasian Journal of Information Systems, 16, 103-117.

Godinho, M., Fernandes, F., Lima; A. (2009) Pesquisa em gestão da produção na indústria de calçados: revisão, classificação e análise. Gestão de Produção, São Carlos, 16(2), 163-186.

Guoyou, Q., Saixing, Z., Chiming, T. Haitao, Y. Hailang, Z. (2011) Stakeholders' influences on corporate green innovation strategy: a case study of manufacturing firms in China. Corporate Social Responsibility and Environmental Management. (Article in press).

Hailemariam, G., Vom Brocke, J. (2011) What is sustainability in business process management? A theoretical framework and its application in the public sector of Ethiopia. Lecture Notes in Business Information Processing 8th International Workshops and Education Track on Business Process Management, 66, 489-500.

Hammer, M. e Champy, J.A. (1993) Reengineering the corporation: a manifesto for business revolution, Harper Business Books, New York.

Houy, C., Reiter, M., Fettke, P., Loos, P. (2011) Towards Green BPM - Sustainability and resource efficiency through business process management. Lecture Notes in Business Information 
Processing. 8th International Workshops and Education Track on Business Process Management, $66,501-510$.

Jabbour, C.J.C. (2010) In the eye of the storm: exploring the introduction of environmental issues in the production function in Brazilian companies. International Journal of Production Research, 48(21), 6315-6339.

Kammerer, D. (2009). The effects of customer benefit and regulation on environmental product innovation. Empirical evidence from appliance manufactures in Germany. Ecological Economics, 68(8/9), 2285-2295.

Ko, R., Lee, S. e Lee W. (2009) Business process management (BPM) standards: a survey. Business Process Management Journal, 15.

Kralj, D. (2009) Sytems thinking integrated system approach to sustainable management. 3rd International Conference on Energy and Development - Environment - Biomedicine, 59-64.

Lee, K-H, Kim, J.-W. (2012) Green new product development and supplier involvement: Strategic partnership for green innovation. International Journal of Innovation and Sustainable Development, 6, 290-304.

Paunescu, C., Acatrinei, C. (2012) Managing maturity in process-based improvement organizations: a perspective of the Romanian companies. Journal of Business Economics and Management, 13, 223-241.

Psomas, E. L., Fotopoulos, C. V., Kafetzopoulos, D. P. (2011) Core process management practices, quality tools and quality improvement in ISO 9001 certified manufacturing companies. Business Process Management Journal, 17, 437-460.

Rennings, K. (2000) Redefining innovation - Eco-innovation research and the contribution from ecological economics" Ecological Economics,32, 319-332.

Schiederig, T. e Tietze, F. (2012) Green innovation in technology and innovation management - an exploratory literature review ( Review ) R and D Management, 42, 180-192.

Schonberger, R. (1986) World class manufacturing: the lessons of simplicity applied.

Smith, H., Fingar, P. (2003) Business process management - the third wave: business process modelling language (bpml) and its pi-calculus foundations. Information and Software Technology, 45, 1065-1069.

Stalk, G., Evans, P. e Shulman, L. (1992) Competing on capabilities: the new rules of corporate strategy. Harvard Business Review, 57-69.

Tseng, M-L., Wang, R., Chiu, A.S.F., Geng, Y., Lin, Y.H. (2012a) Improving performance of green innovation practices under uncertainty. Journal of Cleaner Production, (Article in press).

Tseng, M., Chiu, A. S. F., Tan, R. R., Siriban-Manalang, A. B. (2012) Sustainable consumption and production for Asia: sustainability through green design and practice. Journal of Cleaner Production. (Article In Press). 
Trkman, P. (2010) The critical success factors of business process management. International Journal of Information Management, 30, 125-134.

Zhu, Q. e Sarkis, J. (2004) Relationships between operational practices and performance among early adopters of green supply chain management practices in Chinese manufacturing enterprises. Journal of Operations Management, 22(3), 265-289.

Data da submissão: 30/11/2012

Data da publicação: 30/08/2013 